\title{
Speed Bump Detection on Roads using Artificial Vision
}

\author{
Ana L. Ballinas-Hernández, Ivan Olmos-Pineda, J. Arturo Olvera-López \\ Benemérita Universidad Autónoma de Puebla, \\ Facultad de Ciencias de la Computación, Puebla, Mexico \\ analuisa.ballinas@correo.buap.mx, \{iolmos, aolvera\}@cs.buap.mx
}

\begin{abstract}
In recent decades, self-driving has been a topic of wide interest for Artificial Intelligence and the Automotive Industry. The irregularities detection on road surfaces is a task with great challenges. In developing countries, it is very common to find un-marked speed bumps on road surfaces which reduce the security and stability of self-driving cars. The existing techniques have not completely solved the speed bump detection without a well-marked signaling. The main contribution of this work is the design of a methodology that use a pre-trained convolutional neural network and supervised automatic classification, by using the analysis of elevations on surfaces through stereo vision, for detect well-marked and no well-marked speed bumps to improve existing techniques.
\end{abstract}

Keywords: Self-Driving Cars, Speed Bump Detection, Artificial Vision, Stereo Vision, Machine Learning.

\section{Introduction}

In recent years, the trend of the automotive industry has been focused on the development of self-driving cars, and several companies have been incorporating technologies aimed at achieving self-driving. One example of this is the cars that park without the need of a human conductor.

Self-driving car competitions have been taking place, such as the DARPA Grand Challenge and the DARPA Urban Challenge, organized by the Defense Advanced Research Projects Agency. In these competitions, cars start from a designated origin with the aim of reaching a specific destination, then crossing diverse extreme environments without a human driver [3]. Derived from these, several research topics in this area have emerged.

In countries like the United States, Japan, Germany, and others, self-driving cars have begun to circulate in trial versions. Self-driving cars have worked properly in environments with acceptable conditions where, through their sensors, they clearly detect road signals and different elements of road traffic. Nevertheless, the circulation of these cars in developing countries has not been possible due to lack of an adequate infrastructure and road signals.

In developing countries, such as Mexico and India, different sorts of irregularities are common on road surfaces. One of such irregularities being the presence 
of high relief speed bumps, which are placed on roads with the purpose of forcing cars to reduce their speed in specific areas, which leads to potential accidents as well as loss of stability. Despite the existence of traffic rules, it is very common to find un-marked speed bumps. The techniques of digital image processing have been focused mainly on the speed bump detection when their traffic signals are well-marked. However, it is difficult to apply these techniques when there is no clear road marking, and therefore some works are being developed to generate algorithms that can address this issue.

This paper presents a methodology for speed bump detection on road surfaces without the need of well-marked signals. The proposal includes a combination of artificial vision techniques and the use of digital cameras. Specifically, digital image processing, stereo vision, machine learning and a convolutional neural network are used to infer whether or not speed bumps are present in the images. Inputs are stereo images of vehicular transit scenarios acquired through digital cameras and the output is the classification of images where it is identified whether or not there are speed bumps.

\section{Related Work}

There are some aspects that have been studied from vehicular transit scenarios such as: identification of streets, lanes, traffic signs and pedestrian crossings, and irregularities on road surfaces such as: garnishes, urban objects, potholes and marked and un-marked speed bumps. This work focuses on the speed bump detection for which some techniques have been applied: digital image processing, monitoring with accelerometers, LIDAR sensors, stereo vision, deep learning and hybrid techniques.

Some methods of digital image processing have been used for the speed bump detection $[5,6,8,32,30]$. Analysis of color patterns and segmentation of interest areas (ROI) for the detection of speed bumps have been used. The main advantage of segmentation algorithms is that they use inexpensive cameras, however, the accuracy in the obstacles detection on surfaces is low, because it is subject to the visualization of obstacle patterns and if the speed bump does not have a well-marked pattern it is not easily detectable. In addition, it is not uncommon not find that there is not a standard regarding the signals correspondent to speed bumps, which complicates the application of the same vision technique to all types of patterns.

Correspondingly, digital image processing has been used in the detection of different vertical traffic signals [11]. Specifically, segmentation algorithms and automatic learning methods are applied for the recognition of preventive sings for speed bumps $[19,16]$. In these works, different aspects that affect the detection are considered as: the environment weather conditions, vision angle, sizes and shapes of the traffic signs. The downside of using these detectors is that the signals do not indicate a precise location of the speed bumps, which causes confusion in the self-driving. 
There are prototypes of mobile devices to monitor road surfaces $[29,17,18$, 13]. These devices capture vibrations by finding irregularities on road surfaces through accelerometers and microcontrollers. They use ultrasonic sensors to detect speed bumps and capture coordinates of these obstacles through GPS (global positioning system). A pattern recognition system has been applied to the accelerometer readings as well as an automatic classifier using support vector machines to recognize anomalies on the surfaces [26]. The advantage of these devices is that they have a very simple design and are inexpensive. However, there are several disadvantages: precision errors are frequent, due to the lack of exactitude of the GPS coordinates, the delay when the system is tested in real time as well as the low accuracy of the sensors.

Another technique studied to detect obstacles on road surfaces is the analysis of data by LIDAR sensors[14,28]. Surfaces have been analyzed to detect the pavement contour and the location of the road where the cars cruise [14]. For this, a parallel algorithm is designed that applies the data filtering of the floor and the objects as well as the segmentation of data using morphological operators. An algorithm for segmenting classical urban objects using digital elevation maps has also been designed [28]. Main disadvantages of these sensors is the high computational cost since large amounts of point clouds that are processed, in addition to that its cost is very high with respect to other sensors.

Some stereo vision techniques for analysing road surfaces have been studied $[23,24,2,1]$. By means of these techniques, a three-dimensional structure of the scene can be obtained from the estimation of $2 \mathrm{D}$ images from different points of view. An algorithm has been designed to detect roads, traffic islands and obstacles by transforming the perceived data into a digital elevation maps, thus making it possible to perceive elevations on the road surface [23]. The main disadvantage is that it is only possible to detect surfaces with a maximum depth of 3 meters and with high heights in the elevations.

Other works can be found, which combine some of the techniques mentioned above with the aim of improving the individual performance of each one. The research carried out by Fernández presents an algorithm that combines the use of medium-cost LIDAR sensors and digital image processing to detect speed bumps as a type of obstacles represented by zebra crosses, typical of Spain and some other Spanish speaking countries [10]. Results from this work reveal that the detection rate of speed bumps is very high. However, when there are many cars with LIDAR sensors, their signals could overlap and become confused.

Figure 1 shows a plot of the numerical results reported in the literature on speed bump detection. As can be seen, most works detect speed bumps with different techniques when their road signals are clearly visible. They present good results but they do not consider the same conditions as this work. There are other works that apply the technique of surface monitoring with accelerometers and other sensors which is a non-predictive technique and it does not correspond to the conditions considered in this work.

There are very few methods that detect speed bumps when their signals are not well-marked. One of the works related to this research is carried out by 
Ana L. Ballinas-Hernández, Ivan Olmos-Pineda, J. Arturo Olvera-López

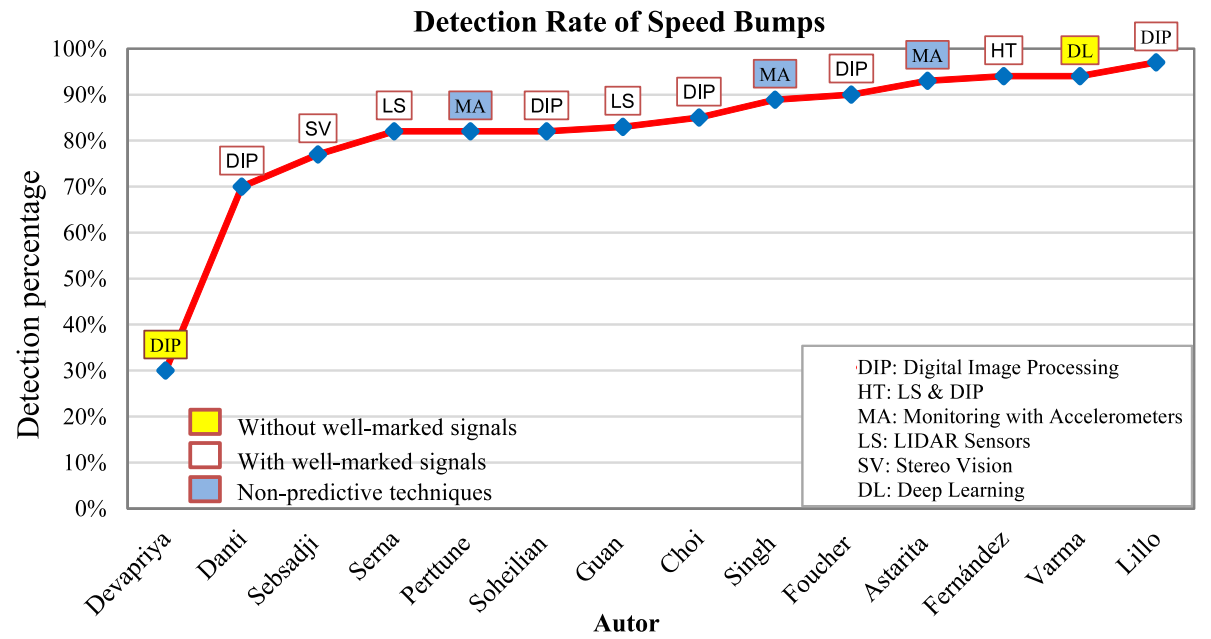

Fig. 1. Speed bump detection rate of different techniques.

Devapriya [8]. Results obtained by Devapriya have a detection rate of $30 \%$ that is below the average as seen in Figure 1. Another work related to the conditions considered in this research is that carried out by Varma [31] in which a method is proposed to detect and inform drivers in real time about the presence of speed bumps with or without signaling. For this purpose, Deep Learning techniques are applied using a pre-trained convolutional neuronal network. The speed bump detection rate is approximately $94 \%$, which is above the average as shown in Figure 1. Still, despite showing good results, no details of the speed bumps can be obtained.

In this work, speed bumps with and without marks are detected and a convolutional neural network is also applied for detection. However, the difference with the work of Varma [31] is that when the convolutional neural network fails, stereo vision is applied to detect speed bumps by analyzing the 3D surface elevation because it is not restricted only to its signaling pattern. In addition, the methodology proposed in this work, unlike Varma [31] and Devapriya [8], would make it possible to identify the kind of speed bumps as well as some other details regarding their shape and size.

\section{Proposed Methodology}

In order to solve the problem of detecting speed bumps on road surfaces, the methodology shown on Figure 2 is proposed. This allows to classify images with or without speed bumps. A convolutional neuronal network is used to detect speed bumps and when these are not easily detected, stereo vision is applied to identify speed bumps by elevation on 3D surfaces. 


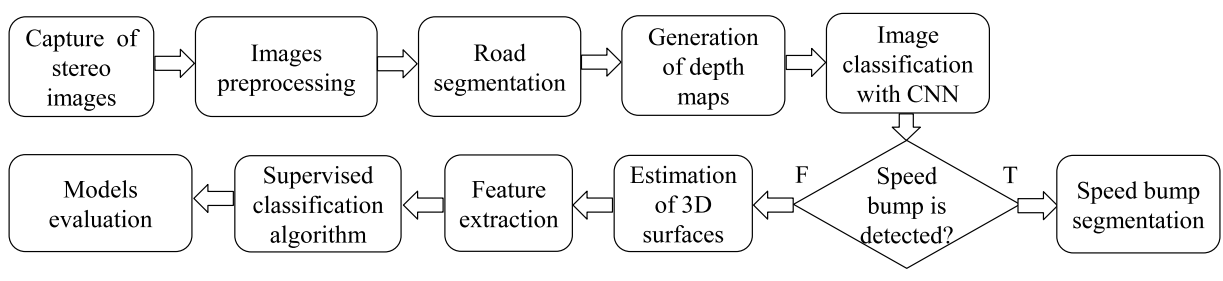

Fig. 2. Proposed methodology for automatic speed bump detection.

\subsection{Capture of Stereo Images}

In this phase a set of stereo images of streets with and without speed bumps is built. The speed bumps types of interest are trapezoidal shaped and pedestrian crossing because they are the most common in developing countries. In addition, the images have marked and un-marked speed bumps road signaling. It is consider environments with controlled conditions like good lighting, preferably without obstacles such as pedestrians and other cars. For capturing the images, a stereo vision system consistent of two cameras with the same characteristics and aligned in parallel with an intersection area between both is used. Streets images of the City of Puebla, Mexico, are captured with and without speed bumps.

\subsection{Image Preprocessing}

This phase consists on the application of digital image processing techniques to improve the quality of the images, thus preparing them for the automatic classification process. The most significant processing techniques required are: lightening the image to increase their clarity and avoiding dark images; decreasing the contrast to reduce the total color range of the image; applying a Gaussian filter to eliminate noise and details of the texture of the street in order to obtain uniform colors or gray intensities [4].

\subsection{Road Segmentation}

The region of interest (ROI) are the streets that will be processed and filtered in order to focus in the area to be assessed. The remaining area is irrelevant and by means of binary masks it is painted in a single color to be ignored in the following stages. A segmentation of color and textures is performed. For color segmentation, the first-order statistical descriptors are calculated considering each pixel: mean, standard deviation, median and entropy. For texture segmentation, second-order statistical descriptors are calculated by using a matrix of co-occurrences considering regions of the image: homogeneity, entropy, dissimilarity, energy and correlation [7]. The image is binarized to generate a mask with the ROI. The ranges of each statistical descriptor are adjusted to generate the mask with the ROI when the descriptor values are within the defined range. 


\subsection{Generation of Depth Maps}

Speed bumps with a well-marked pattern are identified and located. In the event that the image contains not well-marked bumps, the stereo vision is applied to generate depth maps and estimate the 3D surface where the surface elevations can be analyzed in order to find speed bumps. The steps for applying stereo vision to the captured images of the stereo image construction phase are: camera calibration, and rectification of images. The calibration of each camera is performed to find the intrinsic parameters: focal distance, radial and tangential distortion, used to eliminate the distortions of the camera and a stereo calibration is performed to find the extrinsic parameters of both cameras: rotation and translation matrices, used to find the relationship between the coordinates of the cameras with the real world coordinates [12]. Zhans method is one of the most used to calibrate cameras and is based on the detection of corners of chess pattern templates [9]. The aim of the rectification is to make the stereo images exactly parallel to each other horizontally. For this, correspondences between the points of both images are found through rotation and translation transformations until an exact alignment between images is achieved. Subsequently, a disparity map is generated which is a grayscale image where the differences between the pixels of the stereo pair are calculated. A depth map is also generated and the distances to the observed points are estimated.

\subsection{Image Classification Convolutional Neural Network (CNN)}

This allows to classify images with or without speed bumps. A pre-trained model with flat images of a CNN is used to detect speed bumps [25]. If a speed bump is detected with high precision, this is segmented using the depth map and the distance to it is calculated. Otherwise, when speed bumps are not easily detected (when the precision exceeds a threshold) an estimate of 3D surfaces is made to extract characteristics of 3D meshes.

\subsection{Estimation of 3D Surface}

If the CNN cannot distinguish speed bumps, an estimate of the 3D surface is made in order to detect them due to 3D surface elevation. This phase consists on reconstructing a 3D scene by obtaining spatial coordinates and the representation of point clouds relevant to the scene [27]. To generate the disparity maps, the segmented image is used and the stereo vision is applied over the road segmentation. An estimate of 3D surfaces is made by the reconstruction of the three-dimensional scene.

\subsection{Feature Extraction}

An image feature vector that indicates the existence of speed bumps is generated. Features are extracted from both flat images and stereo images. From flat images characteristics of color and textures of speed bumps using low-level and 
shape matching [22]. From stereo images, characteristics are extracted on the 3D meshes estimated as the slope of the elevation, width, height, depth, among others calculated by interpolation functions.

\subsection{Classification Models of Speed Bumps}

For the automatic classification of images, a classifier is trained with a set of flat and stereo image pairs that are labelled in two classes SB and NSB (with and without speed bumps present, respectively). The automatic feature extraction is performed and supervised learning algorithms are applied for the detection of speed bumps such as: decision trees, neural networks, support vector machines, and others, and a predictive model is generated as output [15]. Once trained, the classifier is tested with another set of image pair by applying the generated predictive model to infer the class to which it belongs.

\subsection{Evaluation Models}

The performance of the classifier is measured to determine the combination of features and the classification algorithm that presents the best results. This phase consists on applying evaluation metrics such as: precision, recall, f-measure, and others [15]. To do this, several experiments are carried out with the classifier using different images with and without speed bumps not detected by the CNN. The results of each method are validated to determine which ones show better results. After choosing the model with the best results, when the speed bump is detected by stereo vision it is segmented using the depth map and the its distance is calculated.

When the speed bump is detected, it is segmented and the size, shape, and distance to the speed bump are calculated, since these measures are very important for the decision making of self-driving cars.

This methodology allows to identify a speed bump by two different ways. CNNs represent a good solution for speed bump detection. Visually they work properly when what they find in the images is similar to the instances with which the model was trained. However, the CNN have problems of identification of speed bumps when in their training dataset there are not enough samples similar to the cases that can occur in environments such as the streets of Puebla, Mexico. Thus, stereo vision can promote detection by analyzing the elevations of surfaces when the detection with CNN fails. This proposal can strengthen existing speed bump classifiers.

\section{Preliminary Results}

The expected results of this research work are: the construction of a set of stereo images of roads with and without speed bumps, the implementation of an algorithm for speed bump detection on road surfaces following the methodology proposed, as well as the elaboration of comparative plots of the algorithm results 
to other algorithms. One of the most relevant preliminary results presented in this work is the implementation of the road segmentation.

A set of stereo images with and without speed bumps from the City of Puebla, Mexico, was captured using a stereo vision system built with two equal cameras. It is considered controlled conditions of the environment good climate, lighting, without shadows, and without obstacles on the streets such as pedestrians, cars, among others. These images are preprocessed to improve their quality. A bilateral filter is applied to eliminate noise and to soften the image in order to make the colors more uniform, preserving the edges [4].

A street segmentation algorithm was implemented applying segmentation by color and textures. To achieve this, a dynamic sampling area was defined to calculate a segmentation range. Samples of first-order statistical descriptors are obtained on the HSV and HLS color spaces: mean, median, min, max and the entropy of each channel of the spaces. Simultaneously, second order descriptors are obtained on the RGB space: dissimilarity, homogeneity, energy, correlation, ASM, and entropy, in order to delimit the texture characteristics of speed bumps. From the statistical samples obtained in the images, dynamic intervals are defined to construct a mask that ignores regions outside the range, combining information from each channel. This mask is processed by applying openingclosed filters to reduce the noise of the mask and avoid isolated regions. Results are presented in Figure 3. As can be seen, the region of no interest was removed from the scene and the street was reflected as a region of interest.

Disparity map of stereo images was generated and an example of this is shown in Figure 4a) where the lighter tones indicate that the point is closer and the darker ones are farther away. This map allows to perceive the depth of the scenes, to calculate the distances to different points in it and to reconstruct $3 \mathrm{D}$ scenes. An inicial reconstruction of the three-dimensional scenes was made using point clouds as shown Figure 4b). This Figure shows some key points more representative of the real scene and the depth of them is observed. From the segmented images (Figure 3), a 3D reconstruction of the segmented street surface can be done. In addition, from the key points obtained and appliying mathematical interpolation functions (such as B-splines [21]), it is possible to estimate 3D mesh surfaces. These 3D meshes are inputs to the supervised classifier to detect speed reducers by the analysis of surface elevations when the CNN failure in detection.

Some phases of the methodology have already been implemented. The applied street segmentation techniques have presented visually acceptable results for different street images. In the future, it is expected to calculate the vanishing point of the street to obtain a more precise segmentation of the street, as well as, to implement all phases of methodology: image classification with CNN, estimation of 3D surfaces with B-splines functions, feature extraction of 3D surface meshes, as well as the generation and evaluation of supervised classification models. In addition, it is expected to apply the statistical method of ROC (receiver operating characteristic curve) curves to make a sensitivity and specificity analysis of classification models. 


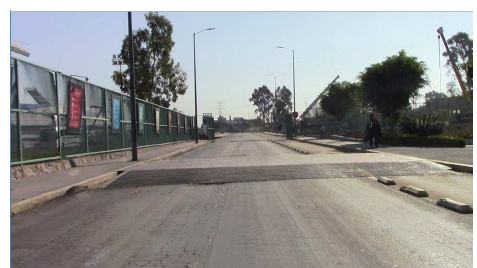

(a)

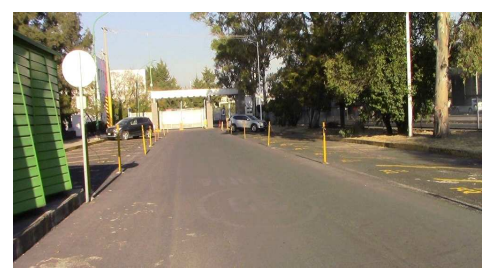

(c)

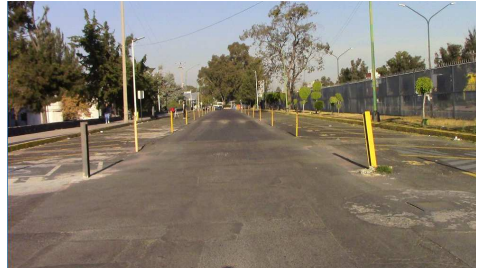

(e)

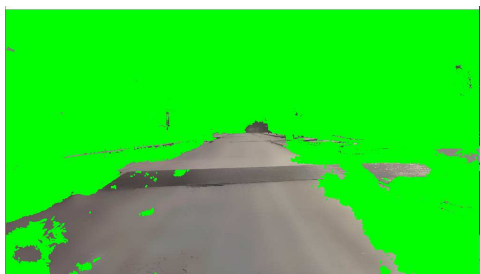

(b)

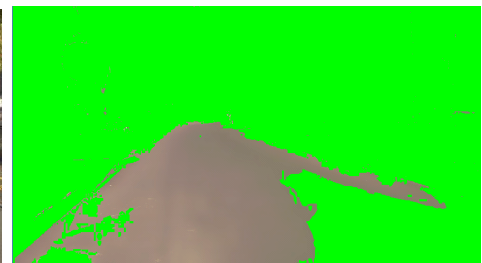

(d)

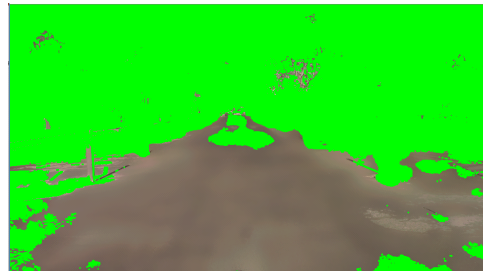

(f)

Fig. 3. Results obtained from street segmentation by color and texture analysis.

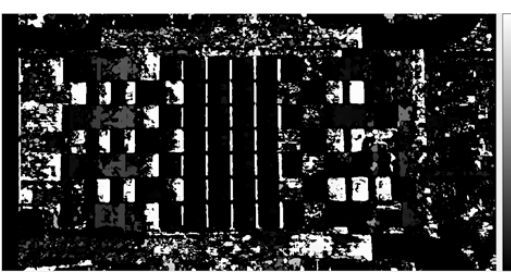

(a)

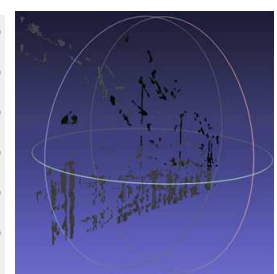

(b)

Fig. 4. a) Disparity map, b)Reconstruction of 3D scene using stereo vision. 


\section{Conclusions}

The detection of speed bumps on roadways is key for a safe and comfortable self-driving, therefore it has been widely studied in the literature, mainly by means of digital image processing techniques. However, speed bump detection without well-marked signals has been little addressed, despite being a very frequent situation in developing countries, and the existing techniques show high errors under this restriction. The main contribution of this work is a methodological proposal for speed bump automatic detection using a pre-trained CNN and supervised automatic classification using a 3D surface estimation obtained by stereo vision without the requirement of well-marked signals, situation that has not been completely solved with existing techniques.

\section{References}

1. Badino, H., Franke, U., Pfeiffer, D.: The stixel world-a compact medium level representation of the 3d-world. In: Joint Pattern Recognition Symposium. pp. 5160. Springer (2009)

2. Broggi, A., Cardarelli, E., Cattani, S., Sabbatelli, M.: Terrain mapping for off-road autonomous ground vehicles using rational b-spline surfaces and stereo vision. In: Intelligent Vehicles Symposium (IV), 2013 IEEE. pp. 648-653. IEEE (2013)

3. Buehler, M., Iagnemma, K., Singh, S.: The DARPA urban challenge: autonomous vehicles in city traffic, vol. 56. springer (2009)

4. Burger, W., Burge, M.J., Burge, M.J., Burge, M.J.: Principles of digital image processing. Springer (2009)

5. Choi, J., Lee, J., Kim, D., Soprani, G., Cerri, P., Broggi, A., Yi, K.: Environmentdetection-and-mapping algorithm for autonomous driving in rural or off-road environment. IEEE Transactions on Intelligent Transportation Systems 13 (2), 974-982 (2012)

6. Danti, A., Kulkarni, J., Hiremath, P.: A technique for bump detection in indian road images using color segmentation and knowledge base object detection. International Journal of Scientific \& Engineering Research 4 (8), 2229-5518 (2013)

7. De Siqueira, F.R., Schwartz, W.R., Pedrini, H.: Multi-scale gray level co-occurrence matrices for texture description. Neurocomputing 120 pp. 336-345 (2013)

8. Devapriya, W., Babu, C.N.K., Srihari, T.: Real time speed bump detection using gaussian filtering and connected component approach. In: Futuristic Trends in Research and Innovation for Social Welfare (Startup Conclave), World Conference on. pp. 1-5. IEEE (2016)

9. de la Escalera, A., Armingol, J.M., Pech, J.L., Gómez, J.J.: Detección automática de un patrón para la calibración de cámaras. Revista Iberoamericana de Automática e Informática Industrial RIAI 7 (4), 83-94 (2010)

10. Fernández, C., Gavilán, M., Llorca, D.F., Parra, I., Quintero, R., Lorente, A.G., Vlacic, L., Sotelo, M.: Free space and speed humps detection using lidar and vision for urban autonomous navigation. In: Intelligent Vehicles Symposium (IV), 2012 IEEE. pp. 698-703. IEEE (2012)

11. Foucher, P., Sebsadji, Y., Tarel, J.P., Charbonnier, P., Nicolle, P.: Detection and recognition of urban road markings using images. In: Intelligent Transportation Systems (ITSC), 2011 14th International IEEE Conference on. pp. 1747-1752. IEEE (2011) 
12. Hamzah, R.A., Ibrahim, H.: Literature survey on stereo vision disparity map algorithms. Journal of Sensors 2016 (2016)

13. Harikrishnan, P., Gopi, V.P.: Vehicle vibration signal processing for road surface monitoring. IEEE Sensors Journal 17 (16), 5192-5197 (2017)

14. Hernández, J., Marcotegui, B.: Filtering of artifacts and pavement segmentation from mobile lidar data. In: ISPRS Workshop Laserscanning 2009 (2009)

15. Hernández Orallo, J., Ferri Ramirez, C., Ramirez Quintana, M.J.: Introducción a la Minería de Datos. Pearson Prentice Hall, (2004)

16. Jung, S., Lee, U., Jung, J., Shim, D.H.: Real-time traffic sign recognition system with deep convolutional neural network. In: Ubiquitous Robots and Ambient Intelligence (URAI), 2016 13th International Conference on. pp. 31-34. IEEE (2016)

17. Madhumathy P, Saurabh Singh, S.S.U.K.: Detection of humps and potholes on roads and notifying the same to the drivers. International Journal of Management and Applied Science 3 (1), 130-133 (2017)

18. Madli, R., Hebbar, S., Pattar, P., Golla, V.: Automatic detection and notification of potholes and humps on roads to aid drivers. IEEE sensors journal 15 (8), 4313$4318(2015)$

19. Maldonado-Bascón, S., Lafuente-Arroyo, S., Gil-Jimenez, P., Gómez-Moreno, H., López-Ferreras, F.: Road-sign detection and recognition based on support vector machines. IEEE transactions on intelligent transportation systems 8 (2), 264-278 (2007)

20. Marrón, B.S.: Co-occurrence matrix and fractal dimension for image segmentation. Revista de Matemática Teoría y Aplicaciones 19 (1), 49-63 (2012)

21. Masud, A., Kannan, R.: B-splines and nurbs based finite element methods for kohn-sham equations. Computer Methods in Applied Mechanics and Engineering 241 pp. $112-127$ (2012)

22. Nixon, M., Aguado, A.S.: Feature extraction and image processing for computer vision. Academic Press (2012)

23. Oniga, F., Nedevschi, S.: Processing dense stereo data using elevation maps: Road surface, traffic isle, and obstacle detection. IEEE Transactions on Vehicular Technology 59 (3), 1172-1182 (2010)

24. Oniga, F., Nedevschi, S.: Curb detection for driving assistance systems: A cubic spline-based approach. In: Intelligent Vehicles Symposium (IV), 2011 IEEE. pp. 945-950. IEEE (2011)

25. Patterson, J., Gibson, A.: Deep learning: A practitioner's approach. " O'Reilly Media, Inc." (2017)

26. Perttunen, M., Mazhelis, O., Cong, F., Kauppila, M., Leppänen, T., Kantola, J., Collin, J., Pirttikangas, S., Haverinen, J., Ristaniemi, T., et al.: Distributed road surface condition monitoring using mobile phones. In: International Conference on Ubiquitous Intelligence and Computing. pp. 64-78. Springer (2011)

27. Revelo, D.A., Usama, F.D., Florez, J.F.: Reconstrucción 3d de escenas mediante un sistema de visión estéreo basado en extracción de características y desarrollado en opencv. Ingeniería y Universidad 16 (2), 485-485 (2012)

28. Serna, A., Marcotegui, B.: Detection, segmentation and classification of $3 \mathrm{~d}$ urban objects using mathematical morphology and supervised learning. ISPRS Journal of Photogrammetry and Remote Sensing 93 pp. 243-255 (2014)

29. Sujitha, M.S., Ramesh, N., Kotamraju, S.K.: Automatic speed controlling of vehicle and detection and notification of potholes and humps. Journal of Engineering and Applied Sciences 100 (9), 1921-1924 (2016) 
Ana L. Ballinas-Hernández, Ivan Olmos-Pineda, J. Arturo Olvera-López

30. Tedeschi, A., Benedetto, F.: A real-time automatic pavement crack and pothole recognition system for mobile android-based devices. Advanced Engineering Informatics 32 pp. 11-25 (2017)

31. Varma, V., Adarsh, S., Ramachandran, K., Nair, B.B.: Real time detection of speed hump/bump and distance estimation with deep learning using gpu and zed stereo camera. Procedia computer science 143 pp. 988-997 (2018)

32. Wang, P., Hu, Y., Dai, Y., Tian, M.: Asphalt pavement pothole detection and segmentation based on wavelet energy field. Mathematical Problems in Engineering 2017 (2017) 